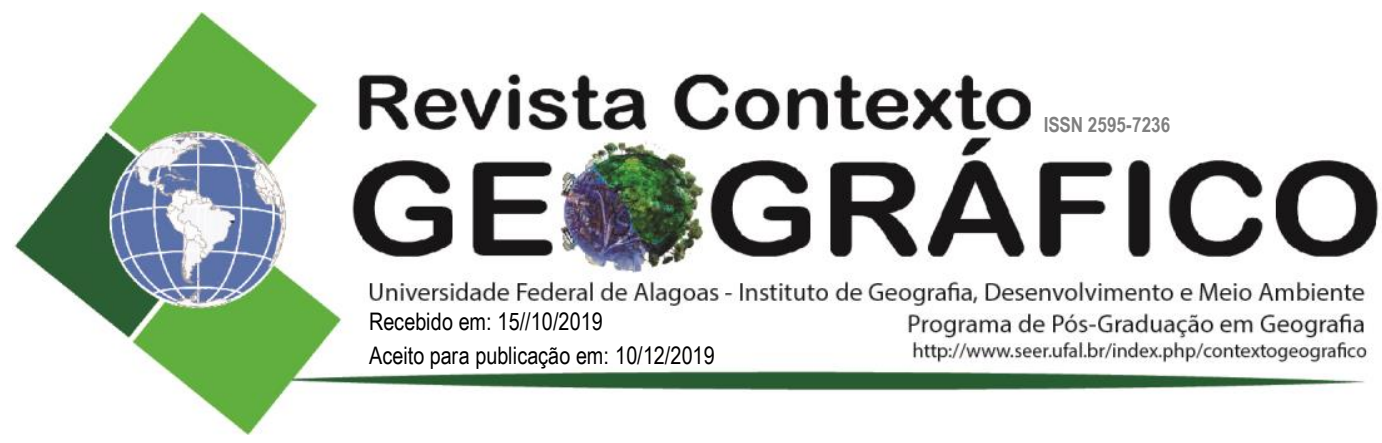

\title{
LA HISTORIA NATURAL DE LA INVESTIGACIÓN EN GEOGRAFÍA HUMANA: LA CONSTRUCCIÓN DEL OBJETO DE INVESTIGACIÓN Y DEL POSICIONAMENTO DE UNA INVESTIGADORA
}

\author{
Flávia Cristine da Silva \\ Grupo de Estudios Geografías Emergentes: políticas, conflictos y alternativas socio- \\ espaciales (GEm), Instituto de Geografía - Facultad de Facultad de Filosofia y Letras - \\ Universidad de Buenos Aires, Argentina \\ flaviacristinedasilva@gmail.com
}

\begin{abstract}
RESUMEN - El objetivo de este artículo es reflexionar sobre la investigación cualitativa en geografía por medio de una narrativa sobre el desarrollo de los procedimientos metodológicos en investigaciones de geografía humana. También se pretende destacar el enfoque etnográfico en la investigación geográfica, además de escribir sobre los giros teóricos y su propio posicionamiento como investigadora, también cómo ha sido construido el objeto de la investigación. Porque la construcción de un posicionamiento como investigadora (así como el objeto de investigación) está lleno de idas y venidas, es un proceso de constante desconstrucción, principalmente sobre saberes, es sobre eso que tratase en este artículo. Este trabajo se basa en experiencias vividas en las investigaciones desarrolladas en la licenciatura, en la maestría y actualmente en el doctorado de la autora.
\end{abstract}

Palabras llaves: Investigación; Geografía Humana; Pobreza Urbana; Vivienda Popular.

\section{A HISTÓRIA NATURAL DA PESQUISA CIENTÍFICA EM GEOGRAFIA HUMANA: A CONSTRUÇÃO DO OBJETO DE PESQUISA E DO POSICIONAMENTO DE UMA PESQUISADORA}

\begin{abstract}
RESUMO - O objetivo deste artigo é refletir sobre a pesquisa qualitativa em geografia por meio de uma narrativa sobre o desenvolvimento dos procedimentos metodológicos em pesquisas de geografia humana. Também pretende-se destacar o enfoque etnográfico na pesquisa geográfica, além de escrever sobre os giros teóricos da pesquisadora e seu próprio posicionamento como pesquisadora, também como tem sido construído o objeto de pesquisa Porque a construção de um posicionamento como pesquisadora (assim como o objeto de investigação) está cheio de idas e voltas, é um processo de constante desconstrução, principalmente sobre saberes, é sobre isto que se trata neste artigo. Este trabalho baseia-se em experiências vividas em pesquisa desenvolvidas ao longa da graduação, mestrado e atualmente no doutorado da autora.
\end{abstract}

Palavras-chaves: Pesquisa científica; Geografia Humana; Pobreza Urbana; Moradia Popular.

\section{THE NATURAL HISTORY IN HUMAN GEOGRAPHY \\ RESEARCH: THE CONSTRUCTION OF THE RESEARCH OBJECT AND THE POSITION OF A RESEARCHER}

\begin{abstract}
The objective of this article to reflect on qualitative research in Geography through a narrative about te development of methodological procedures in human geography research. It is also intended to highlight the ethnographic focus in geographic research, as well as writing about the theoretical turns of the researches and her own positioning as a researcher, also haw the object of the investigation has been created. Because the construction of a
\end{abstract}


researcher position (as the object of research) is full of its comings and goings, it is a constant process of deconstruction, especially about knowledge, and that is what this article. This work is based on experiences lived in the research developed during the bachelor's and master's degree, and currently the doctorate of the author.

Keywords: Research; Human Geography; Urban Poverty; Affordable Housing.

\section{INTRODUCCIÓN}

Este artículo es parte de las reflexiones desarrolladas en el seminario de doctorado "El análisis cualitativo en la investigación" realizado en la Facultad de Filosofía y Letras (FILO) de la Universidad de Buenos Aires (UBA) en el primer cuadrimestre de 2018, bajo la dirección de la Prof ${ }^{a}$ $\mathrm{Dr}^{\mathrm{a}}$ Diana Mazza. Este análisis de la historia natural de la investigación fue guiado por preguntas como: ¿Cómo desarrollaran los procedimientos metodológicos de la autora a lo largo de los años en investigaciones de geografía humana? ¿Qué es lo que lleva la investigadora a investigar esto? ¿Qué dice el objeto de la investigación y la forma en que está construido de su propio posicionamiento como investigadora? Este artículo se suma a reflexiones sobre la metodología en geografía, en especial los diferentes usos del análisis cualitativo y, además, presenta el enfoque etnográfico cómo una herramienta en la operacionalización del concepto de lugar.

\section{EL CAMINAR DE UNA INVESTIGADORA}

En el año de 2004 la investigadora ingresó en la carrera de Geografía en la Universidad Estadual Paulista (UNESP/Brasil) por su interés por las ciudades, en especial para entender la pobreza urbana porque era recién egresa de un técnico en edificaciones y el tema de la vivienda popular y la pobreza urbana fue una constante a lo largo de aquel período. En ese momento la investigadora miraba las ciudades con admiración y se preguntaba ¿Si la humanidad es capaz de construir una cosa tan increíble como las ciudades porqué no es capaz de construir espacios menos desiguales?

En el año 2006 en la UNESP inició su primera investigación, titulada "Segregação socioespacial no Jardín Morada do Sol em Presidente Prudente / SP" (Silva, 2008), con el objetivo de estudiar la segregación socioespacial en las ciudades intermedias. Allí se analizaron las prácticas socioespaciales y el control del territorio, buscando identificar cómo las nuevas formas de producción del espacio acentuaban la tendencia a la segregación, de qué manera los habitantes vivían la misma y cuál era la visión de ellos sobre la ciudad. La pregunta central en este momento de la construcción teórica de la investigadora era ¿Las políticas habitacionales son eficientes para el combate de la pobreza urbana? Esta pregunta fue guiada por la necesidad que las brasileñas y brasileños tienen de presentar una dirección de residencia para lograr la aceptación en un trabajo, o sea, el tema de la vivienda es crucial para que se logre una actividad remunerada formal en el país.

En aquel momento se elaboraron guiones de entrevistas, que se llamaron "entrevistas no dirigidas", o sea, que no poseían una estructura previa. Se trataron de visitas de acercamiento realizadas con algunos líderes del barrio. Es importante destacar esta práctica como fundamental para estudiar pobreza urbana, en cuanto se trata de una "tarjeta de visitas" que llevó 1 la investigadora a establecer contacto con los habitantes con una mínima relación de confianza, lo que posibilitó, entre otras cuestiones, conocer algunos liderazgos en esos espacios. Rockwell (1997) destaca que la forma en que se da el primer contacto marca la experiencia de campo, dado que es importante tener informaciones previas sobre la localidad y también tener representatividad, es decir, haber establecido algunos contactos anteriores que permitan establecer un primer contacto más tranquilo con los entrevistados.

En un según momento de la investigación, la prioridad fueron las entrevistas dirigidas (con un 
guión), fueran echas en dos partes, primero con los líderes del barrio: el presidente de la asociación del barrio, una líder religiosa, la asistente social responsable por el Centro de Referencia de Asistencia Social del barrio, la directora de la única escuela existente en la localidad y la coordinadora de la única entidad filantrópica que actuaba allá. En un segundo momento se entrevistó a una ama de casa, una estudiante, una anciana, un portador de discapacidad física, un desempleado y un comerciante.

\begin{abstract}
"Consideramos a localização de sua residência (áreas de inclusão/exclusão); o poder aquisitivo (faixa salarial de até 05 salários mínimos para os entrevistados representativos de segmentos de menor poder aquisitivo, e uma faixa salarial maior que 05 salários mínimos para os de melhor condição socioeconômica); o meio de deslocamento utilizado (a pé, de bicicleta, de transporte coletivo, de veículo próprio) e alguns tipos sociais definidos por faixa de idade e/ou forma ou não de inserção no mercado de trabalho e/ou condição de vida (idoso, mulher trabalhadora, dona-de-casa, estudante, desempregado, portador de deficiência física e residente em cidade vizinha a Presidente Prudente)". (PEREIRA, 2006, p.26).
\end{abstract}

Como resultado del trabajo de campo, se realizó 5 entrevistas no dirigidas, y entrevistas dirigidas: 5 con líderes del barrio y 6 moradores. El total de las 16 entrevistas resultaran en informes de campo (también llamados notas de campo). Estos fueron estructurados con cierta rigidez, todos conteniendo los ítems: llegada en la institución; perfil de receptividad; descripción del sitio; medio ambiente; y el contenido de las respuestas.

Al final de la investigación se expos cómo las moradoras y moradores vivían y percibían la segregación socioespacial por medio de extractos de las entrevistas realizadas, con el objetivo de "dar voz" a las entrevistadas y entrevistados. La investigadora comprendió que para el combate de la pobreza urbana las políticas habitacionales no bastan por si solas porque la población del barrio estudiado tenía el acceso a la vivienda, pero el tema económico aún era un impedimento para ellos vivieren la ciudad o consumiren/tener acceso a ciertos los espacios urbanos para recreación o compras y hasta para tener acceso a un buen sistema de salud (en este caso, privado); el trabajo informalidad se presenta como una posibilidad de sobrevivencia para la población pobre de las ciudades. Entonces surgieran preguntas centrales: ¿De qué manera los pobres de las ciudades enfrentan el desempleo? ¿Con qué dinero ellos compran los dulces que venden por las calles o compran materiales para invertir en su propia producción? ¿La tarjeta de crédito es una posibilidad de acceder al crédito y empezar una actividad económica?

En 2009 en la Universidad de San Pablo (USP/Brasil) se inició la investigación que después resultó en tesis de maestría "O circuito inferior da economía urbana em Campinas/SP: Análise sobre a mobilidade espacial e o acesso ao crédito" (SILVA, 2012). Allí se analizó el acceso al crédito y las formas de movilidad espacial, basándose en la teoría de Milton Santos (1979) sobre los circuitos de la economía urbana, en especial el circuito inferior de la economía urbana, porque es este circuito que abriga una gran cantidad de trabajadores informales.

Como metodología de investigación se utilizó la diferenciación que el autor hace entre "situaciones geográficas centrales y residenciales". Ese fue el primer criterio para seleccionar las áreas estudiadas en aquella ciudad. Como situación residencial se estudió el distrito de Barão Geraldo (que posee gran número de empresas de alta tecnología y grandes universidades, caracterizado por ser un sub-centro y albergar parte de la clase media del municipio) y el Conjunto Habitacional Vida Nova ubicado en la zona periférica y que alberga parte de la mano de obra trabajadora pobre de la ciudad. Como situación geográfica central estudiamos el Centro Popular de Compras (caracterizado por micro emprendedores individuales y por el trabajo informal) y el Paseo de Compras de la calle 13 de Maio (una peatonal destinada a las compras en general, con 
fuerte presencia de trabajo formal).

Fueron realizadas muchas visitas de aproximación, para establecer contactos; luego, se elaboró una encuesta en concomitancia a la investigación bibliográfica.

Para evaluar este instrumento se hicieron pruebas sobre la encuesta, de forma tal de conocer si los entrevistados entendían las preguntas y modificar lo que fuera necesario, como, por ejemplo, disminuir el tiempo de aplicación porque era demasiado largo (con una duración aproximada de 40 minutos de aplicación). Fueron realizados 3 testes que ayudaron a redirigir la investigación, principalmente para sumar el análisis del fiado como modalidad de crédito (que es la práctica de comprar y poder realizar el pago un tiempo después, esto es un arreglo entre el comerciante y el cliente fundado en la confianza o solidaridad entre las dos personas), lo cual fue una sorpresa muy positiva a la investigación.

Al final de la investigación, fueron realizadas un total de 85 encuestas cerradas con una duración de 15 a 20 minutos cada una, esta opción optimizó el tiempo, pero nos proporcionó un análisis cuantitativo en cuanto a la movilidad urbana y el acceso al crédito en la ciudad de Campinas. Por eso, el interés de profundizar en algunos aspectos referentes al fiado - como la solidaridad orgánica y la confianza que envuelven esta modalidad de crédito - no se pudo alcanzar en un análisis cuantitativo. Cuando se les preguntaba sobre el fiado, los entrevistados inicialmente negaban, pero después de unos minutos de conversación ellos asumían esa práctica alegando la confianza en los clientes antiguos y/o por solidaridad a otros profesionales o a las personas más pobres. El análisis cuantitativo posibilita variables y muestras estadísticas, pero sólo produce "información o conocimiento sobre un plano superficial al alcance de la conciencia de los respondientes" (SIRVENT, 1999, p. 9).

La investigación de maestría propició algunas indagaciones que motivaron el proyecto de doctorado: primero, el interés en analizar los conceptos de confianza y solidaridad orgánica (un conjunto de actividades formado por la existencia común de agentes actuando sobre un territorio común, ella se crea y se alimenta de ofertas del medio geográfico local), porque estos conceptos pueden llevar a la comprensión de la centralidad del lugar para estudiar la pobreza en las grandes ciudades. Por otro lado, Milton Santos ([1979] 2008; 2006) que propuso el uso del concepto de solidaridad orgánica en los estudios geográficos falleció antes de operacionalizarlo, entonces a todos los que estudian su teoría quedó esta tarea con el objetivo de actualizarla. Es importante destacar que esta posibilidad de operacionalización puede indicar caminos a la comprensión del lugar como un punto de partida para lo que Santos (2006) llamó "el período popular de la historia" que es una etapa de desarrollo de la globalización cuando la cultura, la división del trabajo y las relaciones sociales sean direccionadas por "los de abajo" o por los agentes del circuito inferior de la economía, basados en el territorio, la cultura local y las relaciones horizontales y de solidaridad orgánica que se desarrollan en el lugar. Este autor destacó la importancia de estudiar el concepto de lugar, tan rico a la Geografía y la comprensión de la pobreza urbana en los países pobres (SANTOS, 2006).

Sin embargo, el tema de la vivienda volvió cómo central porqué en el análisis de movilidad de los agentes del circuito inferior de la economía urbana se destacó que estos tenían prácticas espaciales muy restrictas al entorno de sus barrios de residencia (en general ubicados en la periferia de la ciudad, para las situaciones geográficas residenciales) o al entorno de sus ubicaciones de trabajo (en el caso de las situaciones geográficas centrales, siendo que la gran mayoría también son moradores de barrios periféricos), en las dos situaciones la justificativa fue el costo con transporte y los largos viajes en colectivo o el tráfico (en caso de utilización de auto particular). Los pobres en la ciudad tienen una baja movilidad porque son forzados a vivir en las periferias de las ciudades, porqué es allá que hay inmuebles con precio accesible de alquiler o de terrenos/inmuebles para comprar.

La dificultad de vivir en el centro de las ciudades y de "consumir la ciudad" es un tema central, es 
la discusión de Lefevbre sobre el "derecho a la ciudad" y los movimientos de lucha por la vivienda muchas veces se ponen en línea de combate frente a especulación y el mercado inmobiliario. Además, ellos promueven espacios mediadores del proceso de busca por respuestas a los interrogantes planteados en el cotidiano (BLOCH, 2007).

Analizar el cotidiano de los movimientos sociales de lucha por la vivienda posibilita operacionalizar los conceptos de lugar, cotidiano, solidaridad orgánica y confianza. De este modo, el proyecto de doctorado "La solidaridad orgánica y la confianza en los movimientos sociales de lucha por la vivienda: la importancia del lugar" está en curso desde el año 2018 en el Instituto de Geografía de la Facultad de Filosofía y Letras Universidad de Buenos Aires (FILO / UBA), bajo dirección de la $\operatorname{Prof}^{a} \operatorname{Dr}^{a}$ Mariana Arzeno (profesora de la UBA) y co-dirección de la $\operatorname{Prof}^{a} \operatorname{Dr}^{a}$ María Mónica Arroyo (profesora y coordinadora del Laboplan - Laboratorio de Geografía Política, Planeamiento Ambiental y Territorial fundado por Milton Santos en la Universidad de São Paulo - USP).

Este proyecto se lleva cabo en los dos países: Brasil y Argentina, se investiga el cotidiano de movimientos de lucha por la vivienda que tienen cómo premisa la autogestión, la ayuda mutua y el cuestionamiento del espacio urbano (sea por luchar por el derecho de los pobres vivieren en el centro de las ciudades o por el "derecho a la belleza", etc. (LAZARINI, 2014). Los objetos de análisis son: en San Pablo un proyecto habitacional ya consolidado el Jardim Apuanã del Movimento dos Sem Terras da Zona Norte y la reciente Ocupación Comandante Almirante Negro de la Frente de Luta por Moradia (FLM). En CABA/Buenos Aires el proyecto habitacional ya consolidado da Cooperativa La Fábrica y en Rosario/ Santa Fe la Cooperativa La Creciente, ambas cooperativas de vivienda que participan del Movimiento de Ocupantes e Inquilinos (MOI).

En esta investigación se propone un análisis del concepto de lugar y, específicamente, la construcción de la solidaridad orgánica y la confianza que surgen de los vínculos la proximidad que se construyen en los lugares. Los interrogantes son ¿Cómo son construidas las relaciones de proximidad adentro de los movimientos sociales de lucha por vivienda? ¿Cuáles son las posibilidades de análisis espaciales de los movimientos de lucha por la vivienda? ¿Las densidades comunicacionales, informacionales y tecnológicas se relacionan con la construcción (o desconstrucción) de la confianza y solidaridad orgánica? ¿Cómo se espacializan la confianza y la solidaridad orgánica (en el sentido de prácticas espaciales) en el lugar (cotidiano)?

En este proyecto el análisis cualitativo permite analizar los significados, valores y emociones que constituyen la vida cotidiana y la interacción humana. El objeto de investigación es construido según los significados que los entrevistados atribuyen a los aspectos del cotidiano. El análisis cualitativo permite construir mapas conceptuales más adecuados a las realidades, entendiendo que la emoción es parte de la construcción del dato científico. Se busca comprender las acciones del individuo o grupo insertados en la totalidad de sus historias y entorno social. La lógica cualitativa:

\footnotetext{
"Está más acerca de enfatizar la inducción analítica y de buscar generación de teoría, la comprensión, la especificidad y las verdades hipotéticas. Es la que habla de trabajar con pocos casos para profundizar el significado que la población otorga al hecho social "(SIRVENT, 1999, p.9).
}

Analizar los datos a partir de la lógica comprensiva, permite que la teoría actúe más como una orientadora qué como una definidora, de modo que se utilize la teoría para analizar y comprender. Este análisis enfatiza la importancia de los interrogantes centrales como un "timón" de la investigación, estos pueden sufrir modificaciones a lo largo de la investigación, pero estas preguntas, aunque sin sentido, son generadoras de sentido.

Cómo metodología de análisis la investigadora adoptó el enfoque etnográfico, que consiste en 
"representar la realidad estudiada, con todas sus diversas capas de significado social en su plena riqueza". Lo que interesa es lo que hay por detrás del cotidiano observado, el punto de vista de los sujetos y la perspectiva con que éste ve a los demás, y eso se puede "percibir en las explicaciones, en las conductas observadas, o pautas susceptibles de sugerir ciertas interpretaciones" (WOODS, 1987, p. 19).

Este enfoque metodológico prima la triangulación observación-participación-entrevista, herramienta fundamental para explorar un problema con mayores probabilidades de exactitud. La observación participante consiste en introducirse en el campo y observar cómo ocurren las cosas en su estado natural. Actualmente la investigadora participa de la Cooperativa de Vivienda La Creciente en la ciudad de Rosario/Santa Fe, vinculada al MOI. Esta práctica permite vivir el cotidiano de la cooperativa y presenciar momentos de decisiones y resoluciones de conflictos en el ámbito colectivo, esto contribuye a la elaboración de preguntas para realización de entrevistas en los demás movimientos sociales investigados.

La confianza para con el investigador es fundamental para el acceso a informaciones honestas y de calidad. Los principales requisitos para la observación es tener "un ojo avizor, un oído fino y una buena memoria", por encima de cualquier método o instrumento de recolección de datos. El investigador es la pieza clave de la investigación, capaz de mezclarse al medio, tomar decisiones, interpretar, etc. (WOODS, 1987, p. 56).

Las entrevistas abiertas sirven para conocer las opiniones y percepciones de las personas sobre los hechos, para oír sus historias y descubrir sus sentimientos. En este momento (similar al de la observación) prestar atención a las palabras, frases o comentarios es fundamental, porque, además de identificar las causas de determinados procesos, pueden indicar la importancia que una cuestión tiene para entrevistado: es la posibilitad de comprensión del sentido común, porque es él que hace el cotidiano, "o senso comum é prática, pode não ser práxis, mas é prática". Este puede ser el camino para "imaginarmos uma vontade transformadora que reúna segmentos sociais com historicidades e espacialidades distintas" (RIBEIRO, 2014, p. 178).

Como en la observación, el objetivo de las entrevistas abiertas es interferir lo menos posible, por lo que las preguntas no están estructuradas, pero pensadas para facilitar la expresión de opiniones y hechos personales con la mayor sinceridad y precisión. En otras palabras, el objetivo es propiciar conversaciones guiadas. Cuanto más natural es el investigador, mayores son las posibilidades de éxito (no conviene presentarse únicamente como investigador, o como un experto en el asunto), es decir relacionarse con las personas, establecer vínculos de persona a persona.

Como se puede percibir, el enfoque etnológico consiste en un análisis cualitativo muchas veces exhaustivo. "Aunque ya se tenga cierta información sobre un tema, tienes que escuchar a varias personas narrar o explicar los mismos sucesos, a fin de poder comprenderlos" sin interrupciones. Eso permite conocer nuevas versiones o detalles del cotidiano (ROCKWELL, 1997, p. 57).

Sobre el trabajo de campo, la práctica de escribir (aunque sea sobre una visita de un área que tal vez el investigador no va seguir investigando al futuro, pero que tenga alguna relación con el objeto de estudio), la investigadora intenta tener el cuidado de realizar un registro en diarios y notas, anotar sus impresiones y sensaciones es un trabajo continuo y personal. Estas anotaciones, hechas en un momento privado, con reflexiones libres y en el espacio de tiempo más breve posible son importantes para recordar todos los detalles importantes (con posibles notas más extensas escritas posteriormente).

También, el tema de las transcripciones de las entrevistas no puede ser un limitador, estar horas y horas a transcribir totalmente las entrevistas puede ser un factor limitante a la investigación. Para eso, con el enfoque etnológico es posible que se realice la transcripción de solamente partes importantes de las entrevistas, pero se hace necesario escuchar más de una vez las 
grabaciones realizadas porque estas escuchas posibilitan nuevas interpretaciones, que pueden permitir una mayor descripción, más compleja y profunda. Escuchar de nuevo las grabaciones en distintos momentos, posibilita confeccionar índices con los principales contenidos que serán desarrollados en la redacción o puntos que requerirán corrección u orientación. El método etnográfico presupone la alternancia de lectura y escritura, relectura y reescritura, y si es necesario, volver a campo y realizar más entrevistas. Por eso el trabajo de campo, lectura y escrita están juntos en toda la investigación. Es una experiencia muy interesante no postergar el trabajo de campo.

Un punto a destacar es la importancia del debate continuo de ideas con colegas, la participación en reuniones de los grupos de investigación o de grupos de estudios es central para poner "a prueba" sus ideas. También, el proceso de escritura permanente, reordenando de acuerdo con las categorías analíticas utilizadas, pero conservando los detalles particulares, evita la dispersión de información.

\section{CONSIDERACIONES FINALES}

Se hace necesario destacar tres practicas metodológicas que se hicieran (y aún se hacen) fundamental a la investigadora: primero, el momento de acercamiento al objeto de estudio (muchas veces no es registrado o relatado, pero el mismo es parte del trabajo de campo y debe ser registrado y agregado al producto final independiente si el resto del trabajo de campo se desarrolló en este lugar o no), este contacto inicial es fundamental, siendo necesario introducirse como una persona responsable y que lleva a cabo una investigación de valor, y que los entrevistados entiendan la importancia y las contribuciones de la investigación de modo tal de generar empatía y confianza. Esta relación de confianza y respeto se da al insertarse a partir de relaciones con personas particularmente útiles a la investigación (como líderes de movimientos, comerciantes conocidos, líderes religiosos, personas con cierta influencia en la comunidad, etc.); también al estar atento al vestirse, cómo comportarse y qué decir (sus creencias, valores, costumbres, y comunicarse con lenguaje similar), porque tienen un impacto muy grande al momento de insertarse lo más posible en la realidad estudiada (dispuesto a observar y escuchar, más que hablar).

El segundo punto es la elección del tipo de análisis de la investigación (cualitativa o cuantitativa) porque afecta en la elaboración de encuestas abiertas o cerradas. Por ejemplo, podemos caer en el error de querer crear gráficos con datos cualitativos, eso puede empobrecer el análisis. Por otro lado, las preguntas cerradas se adecuan más al análisis cuantitativo.

El tercer punto, es importante realizar la prueba de la encuesta cerrada o del guion de entrevista para probar el instrumento y evitar la pérdida de datos. Un trabajo de campo como un proceso continuo, a lo largo de la investigación, permite el intercambio de informaciones prácticas (campo) y teóricas (lecturas), es importante esta concomitancia. En cualquier tipo de análisis es un peligro la realización del trabajo del campo al fin de la investigación, esa práctica puede traer sorpresas desagradables y evitar un análisis más profundo de los datos a la luz de la teoría, también puede impedir o dificultar avances teóricos por falta de tiempo.

Cuanto al objeto de la investigación, un tema que empezó con cuestionamientos de una alumna de nivel técnico, llevó la investigadora a actualmente pensar la cuestión habitacional no como una "crisis", pero sí, la vivienda como una mercadoría (como cualquier otra) cómo presenta Engels en sus escritos sobre el problema de la vivienda. Y, por otro lado, pensar la cuestión de la vivienda involucrada con el "habitar" como posibilidad de adentrar el cotidiano y el concepto de lugar - tan rico a la geografía. (DE CERTEAU; GIARD; MAYOL, 1999; MARTÍNEZ, 2014).

La construcción de un posicionamiento como investigadora (así como el interés de 
investigación) está lleno de idas y venidas, es un proceso de constante desconstrucción, principalmente sobre prejuicios y saberes (uno no detiene mayor sabiduría que el otro, solamente detiene una sabiduría diferente). La dificultad es de construir una mirada al mundo que saiga desde un lugar de privilegios a ponerse de manera a mirar el mundo desde la mirada del otro. También poner las ideas en diferentes espacios de discusión, principalmente donde hay divergencias de pensamiento, porque la diferencia es una gran fuerza creativa, pero es difícil porque fuerza a uno que dialogue con ideas contrarias y eso exige mucha energía y abertura al diálogo. Es decir, uno tiene que estar dispuesto a escuchar, más do que hablar - como un ejercicio permanente, con errores y aciertos.

\section{REFERENCIAS}

BLOCH, J. A. (2007). O direito à moradia: um estudo dos movimentos de luta pela moradia no centro de São Paulo. Tesis de maestría en Sociología -Facultad de Filosofía, Letras y Ciencias Humanas de la Universidad de São Paulo, São Paulo.

DE CERTEAU, M.; GIARD, L.; MAYOL, P. (1999). La invención del cotidiano 2 - habitar, cocinar. México: Universidad Iberoamericana. (Capitulo 1: el barrio).

LAZARINI, K. (2014). Luta por moradia e autogestão em Buenos Aires: da crise à construção popular do habitat. Tesis de maestría en Geografía - Facultad de Arquitectura y Urbanismo, Universidad de São Paulo, São Paulo.

MARTÍNEZ, E. (2014). Configuración urbana, hábitat y apropiación del espacio. In: Revista Electrónica de Geografía y Ciencias Sociales. Universidad de Barcelona, vol. XVIII, n. 493(33), 1 de noviembre de 2014.

PEREIRA, S. R. (2006). Percursos urbanos: mobilidade espacial, acessibilidade e o direito à cidade. Tesis de Doctoramiento en Geografía - Facultad de Ciencias y Tecnología, Universidad Estadual Paulista, Presidente Prudente.

SANTOS, M. ([1979] 2008). O Espaço dividido: os dois circuitos da economia urbana dos países subdesenvolvidos. 2. ed., São Paulo: Editora da Universidade de São Paulo.

(2006). Por Uma Outra Globalização: do Pensamento Único a Uma Consciência Universal. $13^{\mathrm{a}}$ ed. Rio de Janeiro: Record.

SARLÉ, P. La historia natural de la investigación cualitativa. Revista del Instituto de Investigaciones en Ciencias de la Educación. Año XI, Nº 21. Pp. 25-30.

SILVA, F. C. (2008). Segregação Socioespacial no Jardim Morada do Sol em Presidente Prudente/SP. Tesis de licenciatura en Geografía. Facultad de Ciencias y Tecnología de la Universidad Estadual Paulista, Presidente Prudente.

(2012). O circuito inferior da economia urbana em Campinas/SP: análise sobre a mobilidade espacial e o acesso ao crédito. Tesis de maestría en Geografía. Facultad de Filosofía, Letras y Ciencias Humanas de la Universidad de São Paulo, São Paulo.

SIRVENT, M. T. (1999) Los diferentes modos de operar en investigación social. Observación y Estadística Educacional I. Ficha de Cátedra. Buenos Aires. OPFyL, F.F. y L., UBA.

ROCKWELL, E. (2009). La experiencia etnográfica. Historia y cultura en los procesos educativos. Buenos Aires, Paidós.

WOODS, P. (1987). La escuela por dentro. La etnografía en la investigación educativa. Madrid, Centro de Publicaciones del MEC y Ediciones Paidós Ibérica. 